\title{
Interactions of chiral two-forms
}

\author{
Xavier Bekaert* \\ Physique Théorique et Mathématique \\ Université Libre de Bruxelles \\ Campus Plaine C.P. 231 \\ B-1050 Bruxelles, Belgium - . \\ E-mail: 'xbekaert@ulb.ac.be!
}

ABstract: Two issues regarding the interactions of the chiral two-forms are reviewed. First, the problem of constructing Lorentz-invariant self-couplings of a single chiral two-form is investigated in the light of the Dirac-Schwinger condition on the energy-momentum tensor commutation relations. We show how the Perry-Schwarz condition follows from the Dirac-Schwinger criterion and point out that consistency of the gravitational coupling is automatic. Secondly, we study the possible local deformations of chiral two-forms. This problem reduces to the study of the local BRST cohomological group at ghost number zero. We proof that the only consistent deformations of a system of free chiral two-forms are (up to redefinitions) deformations that do not modify the abelian gauge symmetries of the free theory. The consequence of this result for a system consisting of a number of parallel M5-branes is explained.

\section{Introduction}

In the limit where bulk gravity decouples, the M5-brane is described by a six-dimensional field theory. Its bosonic sector contains, besides the five scalar fields which describe the position of the brane in transverse space, a chiral two-form (i.e. a two-form the field strength of which is self dual).

A single M5-brane with strong classical fields is well understood; its Lagrangian is described in 1.1. For a long time, the obstacle to get such an action was due to the presence of the chiral two-form. When one tries to tries to incorporate the self-duality condition into an action a problem arises with preserving Lorentz invariance. There is a simple non-manifestly Lorentzinvariant free Lagrangian which has been given in [2], and which generalizes the Lagrangian of [3i] for chiral bosons (the couplings to gravitation are included). As a non linear generalization, a Born-Infeld-like action for for a self-interacting chiral two-form was proposed in [4]- The couplings to gravitation were added in a second pa-

\footnotetext{
*From joint work with M. Henneaux and A. Sevrin.
}

per [05]. A manifestly covariant formulation of chiral $p$-forms has been developed in an interesting series of papers [i, characterized by the presence of an extra field and an extra gauge invariance. This extra field occurs non-polynomially in the action, even for the free chiral $p$-forms. It has been shown to be equivalent to the non-manifestly covariant treat-

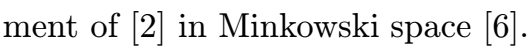

The question of Lorentz-invariant self-couplings (as well as consistent self-couplings in an external gravitational background) for a single chiral 2 -form is the subject of the section that this question can be handled by means of the Dirac-Schwinger condition on the commutation relations of the components of the energymomentum tensor $[\bar{i} \overline{1}]$. This condition leads directly to the differential equation obtained in $\left[\begin{array}{l}{[4} \\ -1\end{array}\right]$ and implies automatically consistency of the gravitational coupling [iأin

When several, say $n$, M5-branes coincide, little is known ${ }^{1}$. Compactifying one dimension of

\footnotetext{
${ }^{1}$ There are several indications that this is a highly unusual system. Both entropy considerations [9 90.$]$ and the cal-
} 
M-theory on a circle yields type IIA string theory. If the branes are longitudinal to the compact direction, the M5-branes appear as a set of coinciding D4-branes which are quite well understood. Their dynamics is governed by a five-dimensional $U(n)$ Born-Infeld theory which, ignoring higher derivative terms, is an ordinary $U(n)$ non-abelian gauge theory. Turning back to the eleven dimensional picture, this suggests that a non-abelian extension of the chiral two-form should exist.

Deformations of a system of $N$ free chiral two-forms in six dimensions are only considered ( $N$ is expected to grow like $n^{3}$ ). We ignore the fermions and the scalar fields as we believe that they will not modify our conclusions ${ }^{2}$. The analysis, outlined in the section $\bar{p}$, shows that all continuous and local deformations of the free chiral 2 -forms does not modify the gauge algebra which remains abelian [i1 in. As a consequence, no local field theory continuously connected with the free theory can describe a system of $n$ coinciding M5-branes. This does not exclude non-local deformations of the abelian theory or local lagrangians that cannot be continuously deformed to the free one.

\section{Covariant self interactions of a sin- gle chiral two-form}

The non manifestly covariant action for a chiral 2 -form in a gravitational background is

$$
S\left[A_{i j}\right]=\int d x^{0} d^{5} x B^{i j} \partial_{0} A_{i j}-\int d x^{0} H
$$

with

$B^{i j}=\frac{1}{3 !} \epsilon^{i j k l m} F_{k l m} \quad F_{\mu \nu \lambda}=\partial_{\mu} A_{\nu \lambda}-\partial_{\nu} A_{\mu \lambda}-\partial_{\lambda} A_{\nu \mu}$

and

$$
H=\int d^{5} x\left(N \mathcal{H}+N^{k} \mathcal{H}_{k}\right)
$$

The spatial indices are denoted by latin indice and go from 1 to 5. $N$ and $N^{k}$ are the standard

culation of the conformal anomaly of the partition function [10] show that the theory should have $n^{3}$ instead of $n^{2}$ degrees of freedom.

${ }^{2}$ In fact this can easily be proven for the scalar fields because they are inert under the two-form gauge symmetry. lapse and shift. The magnetic field $B^{i j}$ is a spatial tensor density of weight one. In the absence of self-interactions, the energy density $\mathcal{H}$ is given by [i:

$$
\mathcal{H}=\frac{1}{\sqrt{g}} B^{i j} B_{i j}
$$

where the spatial indices are lowered and raised with the spatial metric and its five-dimensional inverse, while $g$ is the determinant of $g_{i j}$. The energy density generates displacements normal to the slices of constant $x^{0}$. The momentum density $\mathcal{H}_{k}$, on the other hand, is purely kinematical and generates tangent displacements. It is explicitly given by

$$
\mathcal{H}_{k}=\frac{1}{2} \epsilon_{i j m n k} B^{i j} B^{m n} .
$$

In order to write the action $(2.1)$, it is necessary to assume that spacetime has the product form $T \times \Sigma$ where $T$ is the manifold of the time variable (usually a line). Of course, a spatial coordinate could equivalently play the rôle of the time variable, as in [4i $]$.

Since $B^{i j}$ is gauge-invariant and identically transverse $\left(\partial_{i} B^{i j}=0\right)$, the action (2.1) is manifestly invariant under the usual gauge transformations $\delta_{\Lambda} A_{i j}=\partial_{i} \Lambda_{j}-\partial_{j} \Lambda_{i}$. In flat space, it is also invariant under Lorentz transformations, but these do not take the usual form [2].

We define the exterior form $B$ to be the (timedependent) spatial 2-form with components $B_{i j} / \sqrt{g}$. The equations of motion that follow from the action are

$$
\tilde{d}[N(E-B)]=0,
$$

where $E$ is the electric spatial 2-form defined through

$$
E_{i j} \equiv \frac{\dot{A}_{i j}-N^{s} F_{s i j}}{N}
$$

and where $\tilde{d}$ is the spatial exterior derivative operator. In the case where the second Betti number $b_{2}(\Sigma)$ of the spatial sections vanishes, this equation implies $N(E-B)=\tilde{d} m$, where $m$ is an arbitrary spatial 1 -form. To bring this equation to a more familiar form, one sets $m_{i}=A_{0 i}$. The equations of motion read then

$$
F={ }^{*} F \text {, }
$$

where $F_{0 i j}=\dot{A}_{i j}-\partial_{i} A_{0 j}+\partial_{j} A_{0 i}$. This is the standard self-duality condition. Alternatively, one 
may use the gauge freedom to set $m=0$, which yields the self-duality condition in the temporal gauge $(E=B)$.

The brackets of the gauge-invariant magnetic fields $B^{i j}$ can be found by follow the Dirac method for constrained systems [1 $12 \overline{2}$ ] but one may shortcut the whole procedure and directly read the brackets from the kinetic term of the action (2.1)

$$
\left[B^{i j}(\mathbf{x}), B^{m n}\left(\mathbf{x}^{\prime}\right)\right]=\frac{1}{4} \epsilon^{i j m n k} \delta,_{k}\left(\mathbf{x}-\mathbf{x}^{\prime}\right) .
$$

A direct calculation of the brackets of the energy densities $\mathcal{H}(\mathbf{x})$ at two different space points using only the form of $\mathcal{H}$ and the brackets (2.9) yields

$$
\left[\mathcal{H}(\mathbf{x}), \mathcal{H}\left(\mathbf{x}^{\prime}\right)\right]=\left(\mathcal{H}^{k}(\mathbf{x})+\mathcal{H}^{k}\left(\mathbf{x}^{\prime}\right)\right) \delta,_{k}\left(\mathbf{x}-\mathbf{x}^{\prime}\right) .
$$

Now, we can ask ourselves how can we introduce interactions preserving Lorentz invariance? When one can use the tensor calculus, it is rather easy to construct interactions that preserve Lorentz invariance ${ }^{3}$. But there is an alternative way to control Lorentz invariance. It is through the commutation relations of the energymomentum tensor components. Because the energy momentum tensor is the source of the gravitational field, the method gives at little extra price a complete grasp on the gravitational interactions. As shown by Dirac and Schwinger [ī], a sufficient condition for a manifestly rotation and translation invariant theory (in space) to be also Lorentz-invariant is that its energy density fulfills the commutation relations $\left(\overline{2} \cdot \overline{1} 0_{1}^{\prime}\right)$. The condition is necessary when one turns to gravitation [1] 13 ] The method is more cumbersome than the tensor calculus when one can use the tensor calculus, but has the advantage of being still available even when manifestly invariant methods do not exist.

In the Dirac-Schwinger approach, the question is to find the most general $\mathcal{H}$ fulfilling $(2.10 .10$. The energy-density $\mathcal{H}$ must be a spatial scalar density in order to fulfill the kinematical commutation relations $\left[\mathcal{H}(\mathbf{x}), \mathcal{H}_{k}\left(\mathbf{x}^{\prime}\right)\right] \sim \mathcal{H}\left(\mathbf{x}^{\prime}\right) \delta,{ }_{k}(\mathbf{x}-$ $\mathbf{x}^{\prime}$ ) and depends on $A_{i j}$ through $B_{i j}$ in order

\footnotetext{
${ }^{3}$ These interactions should also preserve the number of (possibly deformed) gauge symmetries (if any), but this aspect is rather immediate for $p$-form gauge symmetries although it is less obvious for the extra gauge symmetry of 6 .
}

to be gauge-invariant. In five dimensions, there are only two independent invariants that can be made out of $B_{i j}$, e.g.

$$
y_{1}=-\frac{1}{2 g} B_{i j} B^{i j}, \quad y_{2}=\frac{1}{4 g^{2}} B_{i j} B^{j k} B_{k m} B^{m i} \text {. }
$$

Set

$$
\mathcal{H}=f\left(y_{1}, y_{2}\right) \sqrt{g}, f_{1}=\partial_{1} f, f_{2}=\partial_{2} f
$$

Then, the condition $\left(\overline{2} . \overline{1} 0^{\prime}\right)$ yields to

$$
f_{1}^{2}+y_{1} f_{1} f_{2}+\left(\frac{1}{2} y_{1}^{2}-y_{2}\right) f_{2}^{2}=4
$$

which is precisely the equation found in [4]. The Dirac-Schwinger criterion yields thus directly the Perry-Schwarz equation, whose solutions are in-

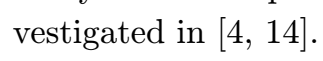

In the flat space context $\left(g_{i j}=\delta_{i j}, N=1\right.$, $\left.N^{k}=0\right)$, the equation $\left(2.10^{\prime}\right)$ guarantees that the interactions are Lorentz-invariant and no further work is required. It also guarantees complete consistency in a gravitational background because of locality of $\mathcal{H}$ in the metric $g_{i j}$.

\section{Consistent deformations of free chi- ral two-forms}

The starting point is the action of a collection of $N$ free chiral 2-forms $A_{i j}^{A}(A=1, \ldots, N)$ in Minkowski spacetime

$$
S\left[A_{i j}^{A}\right]=\sum_{A} \int d^{6} x\left(B^{A i j} \dot{A}_{i j}^{A}-B^{A i j} B_{i j}^{A}\right)
$$

which is the sum of $N$ free action of the form (2.1) in flat background. The magnetic fields $B^{A i j}$ in (3.1) are defined through

$B_{i j}^{A}=\frac{1}{3 !} \epsilon_{i j k l m} F^{A k l m}, F_{i j k}^{A}=\partial_{i} A_{j k}^{A}+\partial_{j} A_{k i}^{A}+\partial_{k} A_{i j}^{A}$.

The action $S_{0}$ is invariant under the following abelian gauge transformations

$$
\delta_{\Lambda} A_{i j}^{A}=\partial_{i} \Lambda_{j}^{A}-\partial_{j} \Lambda_{i}^{A} .
$$

This set of gauge transformations is reducible because if $\Lambda_{i}^{A}=\partial_{i} \epsilon^{A}$, the variation of $A_{i j}^{A}$ vanishes.

Our strategy for studying the possible local deformations of the action (3.1) is based on the 
observation that these are in bijective correpondence with the local BRST cohomological group $H^{0,6}(s \mid d)$ [1] acting on the fields, the ghosts, and their conjugate antifields, $d$ is the ordinary space-time exterior derivative and the upper indices refer to ghost number and form degree resp. By applying the standard method of the antifield formalism [1흐. ghosts of ghosts $\eta^{A}$, and the antifields $A^{* A i j}$, $C^{* A i}$ and $\eta^{* A}$. Their respective parity, ghost number, antighost number are listed in following table

\begin{tabular}{|c|c|c|c|c|}
\hline & parity & antigh & puregh & gh \\
\hline$A_{i j}^{A}$ & 0 & 0 & 0 & 0 \\
\hline$C_{i}^{A}$ & 1 & 0 & 1 & 1 \\
\hline$\eta^{A}$ & 0 & 0 & 2 & 2 \\
\hline$A^{* A i j}$ & 1 & 1 & 0 & -1 \\
\hline$C^{* A i}$ & 0 & 2 & 0 & -2 \\
\hline$\eta^{* A}$ & 1 & 3 & 0 & -3 \\
\hline$s$ & 1 & - & - & 1 \\
\hline$\delta$ & 1 & -1 & 0 & 1 \\
\hline$\gamma$ & 1 & 0 & 1 & 1 \\
\hline
\end{tabular}

Table 1: table of the respective parity, ghost number, antighost number of the variables and the differential operators

The BRST operator $s$ is given by $s=\delta+\gamma$ with

$$
\begin{aligned}
\delta A_{i j}^{A} & =\delta C_{i}^{A}=\delta \eta^{A}=0, \\
\delta A^{* A i j} & =2 \partial_{k} F^{A k i j}-\epsilon^{i j k l m} \partial_{k} \dot{A}_{l m}^{A}, \\
\delta C^{* A i} & =\partial_{j} A^{* A i j}, \quad \delta \eta^{* A}=\partial_{i} C^{* A i}
\end{aligned}
$$

and

$$
\begin{aligned}
\gamma A_{i j}^{A} & =\partial_{i} C_{j}^{A}-\partial_{j} C_{i}^{A}, \\
\gamma C_{i}^{A} & =\partial_{i} \eta^{A}, \quad \gamma \eta^{A}=0, \\
\gamma A^{* A i j} & =\gamma C^{* A i}=\gamma \eta^{* A}=0 .
\end{aligned}
$$

One verifies that $\delta^{2}=\gamma^{2}=\delta \gamma+\gamma \delta=0$.

The cocycle condition defining elements of $H^{0,6}(s \mid d)$ is the "Wess-Zumino condition" at ghost number zero,

$$
s a+d b=0, \quad g h(a)=0 .
$$

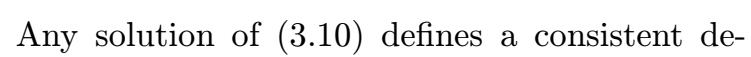
formation of the action (3.1) through $S\left[A_{i j}^{A}\right] \rightarrow$
$S\left[A_{i j}^{A}\right]+g \int d^{6} x a_{0}$, where $a_{0}$ is the antifield-independent component of $a$. The deformation is consistent to first-order in $g$, in the sense that one can simultaneously deform the original gauge symmetry (3.3) in such a way that the deformed action is invariant under the deformed gauge symmetry up to terms of order $g$ (included). The antifield-dependent components of $a$ contain informations about the deformation of the gauge symmetry. Trivial solutions of $\left(\bar{p} . \overline{1} \overline{0}_{1}\right)$ are of the form $a=\gamma c+d e$ and correspond to $a_{0}$ 's that can be redefined away through field redefinitions. Of course, there are also consistency conditions on the deformations arising from higher-order terms $\left(g^{2}\right.$ and higher), but it turns out that in the case at hand, consistency to first order already restricts dramatically the possibilities.

In general, there are three possible types of consistent deformations of the action. First, one may deform the action without modifying the gauge symmetry. In that case, $a$ does not depend on the antifields, $a=a_{0}$. These deformations contain only strictly gauge-invariant terms, i.e., polynomials in the abelian curvatures and their derivatives (Born-Infeld terms are in this category) as well as Chern-Simons terms, which are (off-shell) gauge-invariant under the abelian gauge symmetry up to a total derivative. An example of a Chern-Simons term is given by the kinetic term of (3.11), which can be rewritten as $F \wedge \partial_{0} A^{4}$. Second, one may deform the action and the gauge transformations while keeping their algebra invariant. In BRST terms, the corresponding cocycles involve (non trivially) the antifields $A^{* A i j}$ but not $C^{* A i}$ or $\eta^{* A}$. Finally, one may deform everything, including the gauge algebra; the corresponding cocycles involve all the antifields.

Reformulating the problem of deforming the free action (3.1) in terms of BRST cohomology enables one to use the powerful tools of homological algebra. Following the approach of [1] $\overline{7}_{1}^{1}$, we have completely worked out the BRST cohomogical classes at ghost number zero. In particular, we have established that one can always get rid of the antifields by adding trivial solutions [1] in] (The complete proof will be given in [1] 18$]$ ). This

\footnotetext{
${ }^{4}$ The spatial 2-forms $A_{i j}^{A}$ and their successive time derivatives are effectively independent.
} 
result can be straightforwardly generalized for a system of chiral $p$-forms in $2 p+2$ dimensions $^{5}$. In other words, the only consistent local deformations of a system of free chiral p-forms are (up to redefinitions) deformations that do not modify the abelian gauge symmetries of the free theory. These involve the abelian curvatures or ChernSimons terms. There are no other consistent, local, deformations (This completely justify the assumptions made for the dependence of $\mathcal{H}$ on the $A_{i j}$ through the $B_{i j}$ in the section $\left.\overline{2}_{i}^{1}\right)$.

\section{Acknowledgments}

I am grateful to the organizers for this very enjoyable meeting. I would like to thank my collaborators on the works which were the sources of this talk, M. Henneaux and A. Sevrin. This work has been supported in part by the "Actions de Recherche Concertées" of the "Direction de la Recherche Scientifique - Communauté Française de Belgique" and by IISN - Belgium. The author is "Chercheur I.I.S.N." (Belgium).

\section{References}

[1] P. Pasti, D. Sorokin and M. Tonin, Phys. Lett. B398 (1997) 41, hep-th/9701037; M. Aganagic, J. Park, C. Popescu, J. H. Schwarz, Nucl. Phys. B496 (1997) 191, hep-th/9701166; I. Bandos, K. Lechner, A. Nurmagambetov, P. Pasti, D. Sorokin and M. Tonin, Phys. Rev. Lett. 78 (1997) 4332, hep-th/9701149

[2] M. Henneaux and C. Teitelboim, Phys. Lett. B 206 (1988) 650; M. Henneaux and C. Teitelboim, Consistent Quantum Mechanics of Chiral p-Forms, in Proc. Quantum Mechanics of Fundamental Systems 2, Santiago 1987, Plenum Press (New York 1989).

[3] R. Floreanini and R. Jackiw, Phys. Rev. Lett. 59 (1987) 1873.

[4] M. Perry and J. H. Schwarz, Nucl. Phys. B489 (1997) 47-64, hep-th/9611065

${ }^{5}$ For example, the same techniques have been used in 10 dimensions to show that the only symmetry-deforming consistent vertex for a system of one chiral 4 -form and two 2 -forms is the one that occurs in the type $I I_{B}$ supergravity Lagrangian [19]. These interactions deform the gauge transformations of the exterior forms but not their algebra, which remain abelian.
[5] J. H. Schwarz, Phys. Lett. B395 (1997) 191, hep-th/9701008

[6] P. Pasti, D. Sorokin and M. Tonin, Phys. Lett. B 352 (1995) 59; Phys. Rev. D 52 (1995) 427.

[7] P.A.M. Dirac, Rev. Mod. Phys. 34 (1962) 592; J. Schwinger, Phys. Rev. 130 (1963) 406, 800.

[8] X. Bekaert and M. Henneaux, Int. J. Th. Phys. 38 (1999) 1161, hep-th/9806062

[9] I.R. Klebanov and A.A. Tseytlin, Nucl. Phys. B475 (1996) 164, hep-th/9604089; S.S. Gubser and I.R. Klebanov, Phys. Lett. B413 41, hep-th/9708005

[10] M. Henningson and K. Skenderis, JHEP 9807 (1998) 023, hep-th/9806087

[11] X. Bekaert, M. Henneaux and A. Sevrin, Phys. Lett. B468 (1999) 228, hep-th/9909094

[12] P. A. M. Dirac, Lectures on Quantum Mechanics, Academic Press (New York: 1964); M. Henneaux and C. Teitelboim, Quantization of Gauge Systems, Princeton University Press (Princeton: 1992).

[13] C. Teitelboim, Ann. Phys. (N.Y.) 79 (1973) 542; The Hamiltonian Structure of Spacetime, in General Relativity and Gravitation: One Hundred Years After the Birth of Albert Einstein (A. Held ed.), Plenum Press (New York: 1980); C. Teitelboim, Ph. D Thesis (Princeton: 1973).

[14] M. Hatsuda, K. Kamimura and S. Sekiya, hep-th/9906103.

[15] G. Barnich and M. Henneaux, Phys.Lett. B311 (1993) 123, hep-th/9304057

[16] I.A. Batalin and G.A. Vilkovisky, Phys.Lett. B102 (1981) 27; Phys.Rev. D28 (1983) 2567; Phys.Rev. D30 (1984) 508

[17] G. Barnich, F. Brandt and M. Henneaux, Comm. Math. Phys 174 (1995) 93, hep-th/9405194; M. Henneaux, Phys. Lett. B368 (1996) 83, hep-th/9511145; M. Henneaux, B. Knaepen and C. Schomblond, Comm .Math. Phys. 186 (1997) 137, hep-th/9606181; M. Henneaux and B. Knaepen, Phys. Rev. D56 (1997) 6076, hep-th/9706119; Nucl. Phys. B548 (1999) 491, hep-th/9812140; hep-th/9912052

[18] X. Bekaert, M. Henneaux and A. Sevrin, in preparation

[19] X. Bekaert, M. Henneaux and A. Sevrin, hep-th/9912077 